\section{Coûts de la santé}

\section{Les médecins n'y sont pas indifférents!}

Mais un peu de cohérence s'il vous plait! L'un des défauts majeurs de la LAMal est de vouloir mettre sous le même chapeau concurrence économique et planification, notions par définition antinomiques et source de nombreuses difficultés de fonctionnement.

Loin de lever ces contradictions, la $2^{e}$ partie de la révision de la loi partiellement avalisée par le Conseil des Etats s'enferre encore davantage et se livre à un patchwork difficilement compréhensible sous prétexte de maîtrise des coûts. La révision du financement hospitalier devrait mettre sur pied d'égalité prestations ambulatoires et hospitalières. La vision "moniste" devrait théoriquement permettre de réaliser cet objectif. Mais en transférant en fin de compte la responsabilité de la gestion hospitalière aux caisses-maladie, elle les charge d'une mission fondamentalement nouvelle. Cette option en soi souhaitable parce qu'elle renforcerait la cohésion sociale du système, mais synonyme aussi de nouveau métier et de changement de comportement à quasiment $180^{\circ}$, entre en totale contradiction avec la suppression de l'obligation de contracter.

Cette mesure, sous prétexte de renforcer la concurrence (entre qui? entre les médecins qui travaillent et ceux qui ne travaillent pas?) donne en fait le champ libre aux caisses-maladie pour exercer une pression massive sur l'assurance sociale. Elles pourraient ainsi développer une offre encore plus vaste d'assurances complémentaires de manière à compenser la limitation des prestations et de la qualité. Cette nouvelle source de profit au dépend de l'assurance sociale réjouit les caisses qui pourraient enfin jouer leur rôle d'assureurs à l'américaine. Effrayé des conséquences de son audace, le Conseil des Etats voudrait mettre quelques garde-fous au nouveau pouvoir qu'il leur accorde avec la soi-disant complicité de la FMH. Interviendrait une commission paritaire avec prise en compte d'une clause $d u$ besoin dans la perspective d'un afflux de médecins en raison des bilatérales si par hasard elles étaient enfin ratifiées, du maintien de la reconnaissance du médecin-traitant pour les patients chroniques, de voies de recours, bref un imbroglio de considérations diverses rendant la décision de principe quasiment inopérante et d'une complication administrative extrême.

A cela il faut ajouter, last but not least, le transfert sur les fournisseurs de prestations du contentieux des assurés qui ne paient pas leur prime. Jus$q u$ 'à présent il appartenait aux caisses de maintenir les prestations quitte à entamer une procédure juridique auprès de ces mauvais payeurs avec l'assistance des cantons le cas échéant. Selon la nouvelle mouture, il incomberait désormais aux médecins, aux pharmaciens et aux hôpitaux à la fois d'assumer malgré tout la prise en charge de ces personnes et de procéder aux mesures de recouvrement nécessaires par voie de poursuite ou auprès des services sociaux!

On croit rêver. Le corps médical est conscient de ses responsabilités en matière de santé publique. Il est certes disposé à consentir des sacrifices dans le cadre de TARMED, sacrifices particulièrement conséquents pour certains spécialistes, et même à associer au droit de pratique un contrat portant sur la qualité et l'économicité du traitement de manière à éviter les doublons et lutter contre le tourisme médical. Mais il se refuse à être le dindon de la farce face $\dot{a}$ un paquet de mesures disparates, contradictoires, potentiellement asociales, probablement inefficaces quant à la maîtrise des coûts et même franchement vexatoires.

Dr Yves Guisan

Vice-président FMH, Conseiller national 